\title{
Respuesta temprana de la regeneración de plantas leñosas después del incendio forestal denominado "Las Máquinas" en la Región del Maule, Chile
}

\section{Early response of woody species regeneration after "Las Máquinas" anthropogenic fire in the Maule Region, Chile}

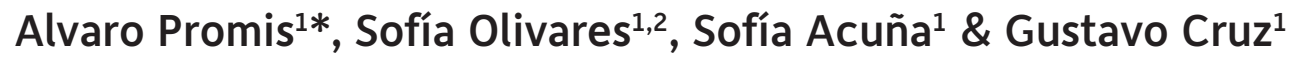 \\ ${ }^{1}$ Departamento de Silvicultura y Conservación de la Naturaleza, Facultad de Ciencias Forestales y de la Conservación de la Naturaleza, Universidad de Chile, Santa Rosa 11315, La \\ Pintana, Santiago, Chile \\ ${ }^{2}$ Fundación Reforestemos, Del Inca 4622, Las Condes, Chile \\ *Email: alvaro.promis@gmail.com
}

The post-fire woody plant species regeneration was assessed in a Nothofagus glauca dominated forest and a clearcutting of Pinus radiata. Native species showed a high vegetative regeneration capacity after wildfire. However, an invasion of seedlings of Pinus radiata is occurring, especially in the N. glauca forest.

Los incendios forestales son un fenómeno mundial, que influyen sobre la composición y estructura de bosques naturales y plantaciones forestales (Castillo et al. 2012, Keeley 2012, Úbeda \& Sarricolea 2016, Thom \& Seidl 2016). En las últimas décadas se ha documentado un incremento en la ocurrencia y severidad de los incendios forestales a escala global (Seidl et al. 2017) y nacional (Úbeda \& Sarricolea 2016), proyectándose un aumento sostenido en el tiempo a causa del cambio climático (González et al. 2011, Seidl et al. 2017).

Por una parte, muchas de las plantas leñosas nativas presentes en los ecosistemas de clima mediterráneo de Chile poseen la capacidad de regenerar vegetativamente (Montenegro et al. 2004) y de tener baja capacidad de regenerar a través de semillas después de un incendio (GómezGonzález et al. 2017). Por otra parte, se ha documentado que los incendios favorecen la regeneración de Pinus radiata D. Don en poblaciones naturales (McDonald \& Laacke 1990), llegando a mostrar un comportamiento invasor en Sudáfrica (Richardson \& Brown 1986) y en Australia post incendio (Williams 2007).

Debido al uso antrópico histórico (quema bosques para uso agrícola, ganadería y sustitución para plantaciones especialmente de $P$. radiata), el bosque natural de la Cordillera de la Costa en la Región del Maule (San Martín \& Donoso 1995) se encuentra fuertemente fragmentado y rodeado por plantaciones forestales (Echeverría et al. 2006), lo que ha favorecido la invasión de $P$. radiata (Bustamante et al. 2003, Gómez et al. 2011). Durante el verano 2016/17, este bosque fue afectado por incendios de gran magnitud, siendo el incendio denominado "Las Máquinas" uno de los de mayor impacto, afectando una superficie de 183.946 ha (CONAF 2017, Valencia et al. 2018). El incendio de Las Máquinas afectó especialmente plantaciones forestales, praderas y matorrales, y bosques naturales de los tipos forestales esclerófilo y caducifolio de Nothofagus obliqua (Mirb.) Oerst. y Nothofagus glauca (Phil.) Krasser (CONAF 2017).

Luego del abandono agrícola ocurrido hacia mediados y fines del siglo pasado, superficies que antiguamente podrían haber recuperado bosques de $N$. glauca fueron ocupadas por formaciones de matorral y bosque, ambos dominados por especies esclerófilas (San Martín \& Donoso 1995, Amigo et al. 2000). En plantaciones de $P$. radiata, después de incendios forestales, la regeneración ocurre principalmente a través de reproducción vegetativa (rebrotes), dando lugar a un matorral compuesto por especies leñosas nativas propias del área (Gómez \& Hahn 2017). La ocurrencia de incendios también favorece la invasión de $P$. radiata al interior de fragmentos de bosques de N. glauca (Litton \& Santelices 2002). Mientras que otros estudios en la Región del Maule han mostrado escasa regeneración de $P$. radiata después de incendios en 
plantaciones de la misma especie y en bosque caducifolio de Nothofagus alessandrii Espinosa (Gómez \& Hahn 2017, Valencia et al. 2018).

El objetivo principal de este trabajo fue evaluar la regeneración de plantas leñosas después de un año de la ocurrencia del incendio "Las Máquinas" en dos situaciones: un bosque dominado por la presencia de N. glauca y en un área con tala rasa de $P$. radiata previa al incendio. Se determinó además los mecanismos de regeneración (vegetativa o sexual) y la estructura de tamaños por especie. Ambas situaciones se encuentran localizadas en la Estación Experimental "Dr. Justo Pastor León", de la Universidad de Chile ( $35^{\circ} 43^{\prime}$ S; $72^{\circ} 29^{\prime}$ O), ubicado a unos dos $\mathrm{km}$ al oeste del poblado de Santa Olga (Fig. 1). En este sector se presenta un clima templado cálido supratermal con régimen de humedad subhúmedo seco. La temperatura media anual del aire es de $13,3{ }^{\circ} \mathrm{C}$, con máxima media de $18,9^{\circ} \mathrm{C}$ y mínima media de $8,9^{\circ} \mathrm{C}$. La precipitación media es de 920 mm/año (AGRIMED 2017). El bosque de N. glauca (BN-Ng; 74,4 ha) presentaba antes del incendio una densidad de 1.627 árb/ha, un área basal de $21,9 \mathrm{~m}^{2} / \mathrm{ha}$ (15,0\% de la densidad y $18,5 \%$ del área basal correspondían a
P. radiata). En el sector de tala rasa (TR-Pr; $22,4 \mathrm{ha}$ ) todos los individuos de $P$. radiata fueron cortados y cosechados el año 2013. Posteriormente en TR-Pr no se volvió a plantar, para que la vegetación se recuperara naturalmente. La severidad del daño causado por el incendio fue clasificada como extrema en BN-Ng y alta en TR-Pr (Castillo et al. 2017). La severidad de daño alta causada en TR-Pr se debió a la reducción de biomasa producida por el incendio sobre la vegetación que se había establecido después de la tala rasa. Ambas superficies seleccionadas se encuentran contiguas. Desde un punto de vista de relieves, las superficies de muestreo presentan pendientes muy suaves a suaves (menos a $30 \%$ de pendiente). Las exposiciones más frecuentes van de este-sureste en BN$\mathrm{Ng}$ y sur-suroeste en TR-Pr.

Para evaluar la regeneración, durante el mes de marzo del año 2018 se estableció una red de parcelas cuadradas de $100 \mathrm{~m}^{2}(10 \times 10 \mathrm{~m})$, aleatoriamente distribuidas. Las parcelas se instalaron con sus lados orientados en dirección a los puntos cardinales. En BN-Ng se establecieron 21 parcelas en una superficie de 5 ha y en TR-Pr se instalaron
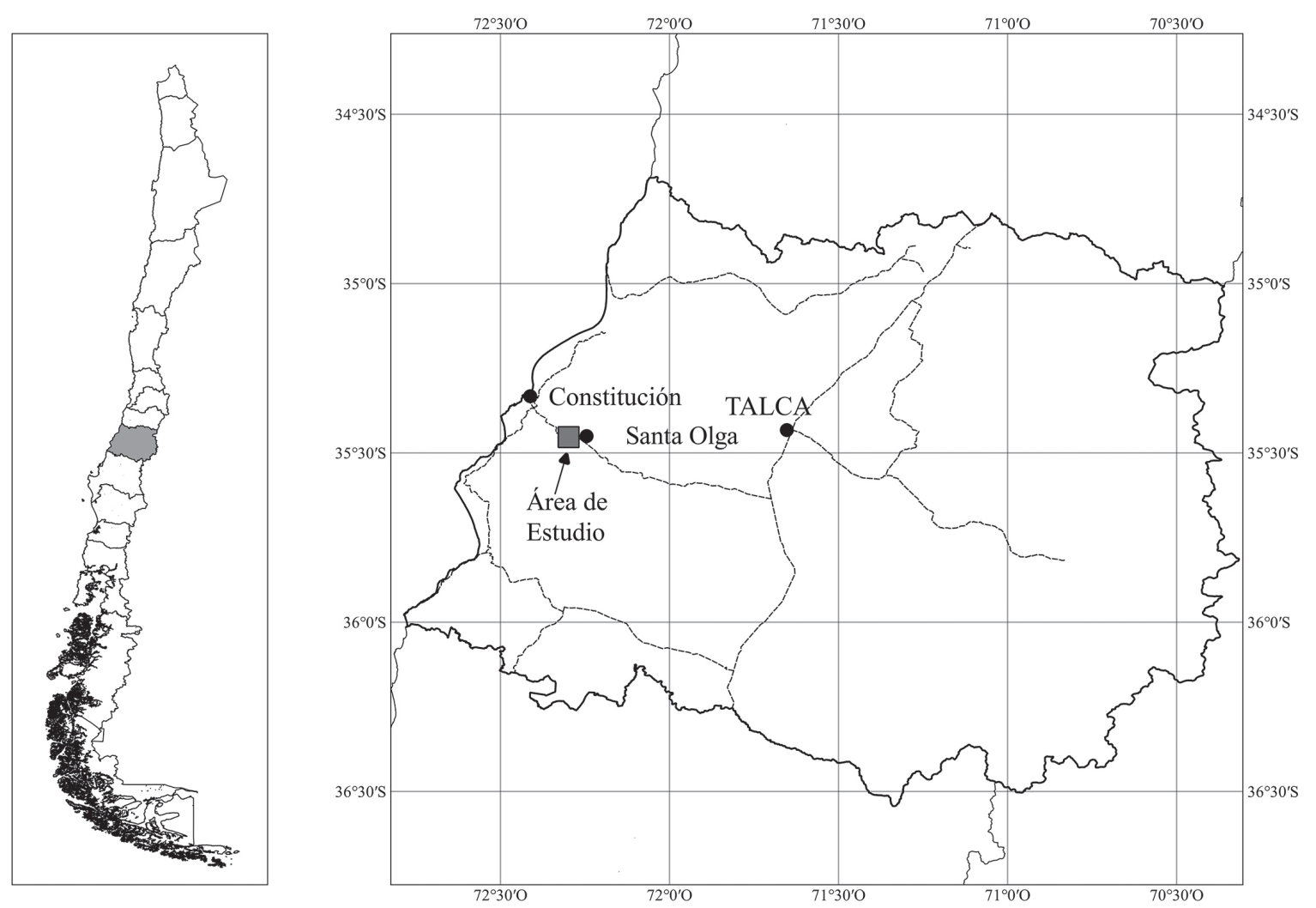

Figura 1. Mapa de Chile y de la Región del Maule mostrando la ubicación del área de estudio en la Estación Experimental Dr. Justo Pastor León, Universidad de Chile (rectángulo gris). / Map of Chile and Maule Region showing the study area in the Experimental Station Dr. Justo Pastor León, Universidad de Chile (grey rectangle). 
42 parcelas en una superficie de 10 ha (esfuerzo de muestreo de 4,2 parcelas por hectárea en ambos casos). Estas parcelas sirvieron para desarrollar un monitoreo de manera permanente. Se determinó la especie, abundancia y la altura de toda la regeneración proveniente de semillas, en cinco subparcelas de $1 \mathrm{~m}^{2}$ en el interior de la parcela. El tamaño de las subparcelas se seleccionó por la alta densidad de plantas de regeneración provenientes de semillas de $P$. radiata. Las subparcelas se ubicaron en los cuatro vértices y una al medio de la parcela. Los resultados se expresaron a través del valor promedio de la especie por parcela. El registro de las plantas que se encontraban regenerando vegetativamente (rebrote) se realizó en una subparcela de $50 \mathrm{~m}^{2}(5 \times 10 \mathrm{~m})$, ubicada hacia la mitad norte de la parcela. Se determinó la especie de todas las plantas leñosas que estaban regenerando vegetativamente, se contó el número de rebrotes por planta y se midió la longitud del más largo en la cepa.

En $\mathrm{BN}-\mathrm{Ng}$ se encontraron 21 especies (distribuidas en 15 familias y 19 géneros) de plantas leñosas regenerando después de un año de ocurrido el incendio, mientras que en TR-Pr se registraron 18 especies (13 familias y 17 géneros), con 15 especies en común (Tabla 1). En ambas situaciones la mayoría de las especies fueron nativas y endémicas, con la presencia de solo dos especies introducidas (Tabla 1). La riqueza de especies en $\mathrm{BN}-\mathrm{Ng}$ (6,6 especies por parcela) fue estadísticamente más alta que la registrada en TR-Pr (4,1 especies por parcela) (prueba $U$ de Mann-Whitney; $U$ $=-2,124 ; p<0,001)$. Del total de especies registradas, 12 especies estaban regenerando exclusivamente por estrategia vegetativa, una especie exclusivamente a través de semilla y 11 especies mostraron ambas estrategias (Tabla 1).

En promedio se establecieron 66.190 plantas de regeneración de semilla por hectárea en $\mathrm{BN}-\mathrm{Ng}$ (rango entre 2.000 y 242.000 plantas/ha). En TR-Pr se establecieron mucho menos, con un promedio de 5.333 plantas de semilla por hectárea (rango desde 0 hasta 32.000 plantas/ha). En ambos ambientes, $P$. radiata es la especie que presenta significativamente mayor cantidad de regeneración por semilla, con un promedio de 64.571 plantas/ha (rango entre 2.000 y 242.000 plantas/ha) en BN-Ng y de 2.143 plantas/ ha (rango entre 0 y 10.000 plantas/ha) en TR-Pr (Tabla 1). La mayoría de las plántulas regenerando por semilla tenían menos de $20 \mathrm{~cm}$ de altura (68,8\% de las plantas en BN-Ng y $75,8 \%$ en TR-Pr) (Tabla 1).

En BN-Ng hay una densidad de 10.610 plantas/ha y en TR-Pr hay 3.664 plantas/ha regenerando vegetativamente. En ambos ambientes, Ugni molinae Turcz. es la especie que muestra mayor cantidad de plantas regenerando vegetativamente $(63,6 \%$ de las plantas en $\mathrm{BN}-\mathrm{Ng}$ y $37,6 \%$ en TR-Pr) (Tabla 1). P. radiata no se encontró regenerando vegetativamente. En BN-Ng las plantas tienen en promedio
10 rebrotes, siendo Cryptocarya alba (Molina) Looser y Quillaja saponaria Molina las especies que se presentan con mayor cantidad de rebrotes (Tabla 1). En TR-Pr las plantas tienen en promedio 8 rebrotes, con el mayor número registrándose en Luma apiculata (DC.) Burret y Teucrium bicolor Sm. (Tabla 1). La longitud promedio ( \pm error estándar) del rebrote más largo por planta después del primer período estival postincendio fue de $72( \pm 13)$ y $93( \pm 20) \mathrm{cm}$ en BN-Ng y Tr-Pr, respectivamente. En BN-Ng y TR-Pr el 77 y $75 \%$ de las especies presentó rebrotes menores a $100 \mathrm{~cm}$ en longitud, respectivamente. En BS-Ng los rebrotes más largos se presentaron en las especies C. alba y N. glauca y en TR-Pr Eucalyptus globulus Labill. y N. glauca (Tabla 1).

Después del incendio la capacidad regenerativa de las especies leñosas fue alta en ambos ambientes, especialmente los arbustos (Montenegro et al. 2004). El matorral arbustivo ha sido señalado por otros autores como una comunidad vegetal que sustituye el bosque costero maulino de N. glauca, por elementos de flora esclerófila (Amigo et al. 2000, Gómez et al. 2009). Sin embargo, la alta presencia de rebrotes de especies de ambientes mesófilos, como U. molinae, N. glauca y Gaultheria insana (Molina) D.J. Middleton, podría llegar a proyectar una mantención del bosque de N. glauca, pero sólo si la regeneración de $P$. radiata no excluye en etapas posteriores a las especies nativas. En TR-Pr la densidad de plantas de regeneración de $P$. radiata fue menor que la de especies nativas, seguramente debido a la inexistencia de árboles en edad reproductiva antes del incendio. La altura de la regeneración vegetativa pudiera prevenir el efecto competitivo de P. radiata, pero se pone en duda que el cierre de copas por el rebrote de N. glauca pueda afectar negativamente la supervivencia de $P$. radiata, por su capacidad de sobrevivir en el sotobosque en competencia por luz (Litton \& Santelices 2002) y por la alta tasa de crecimiento en altura

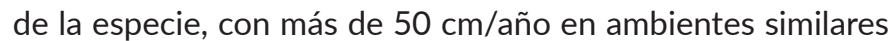
(Rubilar et al. 2008).

Tal como ya ha sido señalado por Litton \& Santelices (2002), la vegetación en bosques de N. glauca ha mostrado una buena capacidad de responder después de la ocurrencia del incendio. Sin embargo, la invasión de $P$. radiata llegará a dominar y transformar estos bosques en la Cordillera de la Costa (Litton \& Santelices 2002, Bustamante et al. 2003). Por lo tanto, en algunos lugares solo medidas de manejo tempranas prevendrán la posible invasión de estos ambientes altamente fragmentados y afectados por los incendios. Un monitoreo permanente sobre estas parcelas permitirá realizar futuros análisis sobre el comportamiento de la vegetación en estos ambientes afectados por incendios, como son el bosque de N. glauca y sectores en los que se promueve la recuperación natural del bosque después de la corta de $P$. radiata a tala rasa. 
TABLA 1. Catálogo de la flora vascular leñosa que se encuentra regenerando después de la ocurrencia de incendio "Las Máquinas" y el número de plantas promedio por hectárea según estrategia de regeneración (semilla o vegetativa), en un bosque dominado por Nothofagus glauca (BN-NG) y una tala rasa de Pinus radiata (TR-PR). Entre paréntesis se presenta el número de rebrotes promedio por planta y la longitud promedio (cm). Letras distintas muestran diferencias significativas (prueba $\mathrm{H}$ de Kruskal-Wallis y prueba U de MannWhitney a posteriori, p > 0,05). La nomenclarura científica de las especies sigue a Rodríguez et al. (2018). / List of the woody vascular plant species of the study area and the mean of the number of individuals per hectare by regeneration strategy (i.e. seeds or vegetative), in a Nothofagus glauca dominated forest (BN-Ng) and a clearcutting of Pinus radiata plantation (TR-Pr) after one year of the occurrence of the "Las Máquinas" wildfire. Numbers in brackets are the average number of resproutings by plant and the average length (cm). Identical letters indicate no significant difference between species for the specific forest and regeneration strategy (Kruskal-Wallis $\mathrm{H}$ test and a post-hoc Mann-Whitney U- test, p > 0.05). Nomenclature follows to Rodríguez et al. (2018).

\begin{tabular}{|c|c|c|c|c|c|c|c|}
\hline \multirow{2}{*}{ Clase / Nombre Científico } & \multirow[t]{2}{*}{ Familia } & \multirow[t]{2}{*}{$\mathrm{Ha}$} & \multirow[t]{2}{*}{ Of } & \multicolumn{2}{|c|}{$\begin{array}{c}\text { Semilla } \\
\text { ( } \mathrm{n}^{\circ} \text { plantas } / \text { ha) }\end{array}$} & \multicolumn{2}{|c|}{$\begin{array}{c}\text { Vegetativa } \\
\text { ( } \mathrm{n}^{\circ} \text { plantas/ha) }\end{array}$} \\
\hline & & & & $\mathrm{BN}-\mathrm{Ng}$ & TR-Pr & $\mathrm{BN}-\mathrm{Ng}$ & TR-Pr \\
\hline \multicolumn{8}{|l|}{ PINOPSIDA } \\
\hline Pinus radiata $\mathrm{D}$. Don & Pinaceae & $A$ & I & $64.571 \mathrm{a}$ & $2.143 \mathrm{a}$ & & \\
\hline \multicolumn{8}{|l|}{ MAGNOLIOPSIDA } \\
\hline Aristotelia chilensis (Molina) Stuntz & Elaeocarpaceae & A & $\mathrm{N}$ & $381 \mathrm{~b}$ & & $29(2 ; 61) b$ & \\
\hline Azara integrifolia Ruiz \& Pav. & Salicaceae & $\operatorname{Ar}$ & $E$ & & $95 \mathrm{c}$ & $495(14 ; 94) b$ & $1.090(12 ; 89) \mathrm{ab}$ \\
\hline Azara lanceolata Hook.f. & Salicaceae & $\operatorname{Ar}$ & $\mathrm{N}$ & & & $10(7 ; 15) b$ & \\
\hline Baccharis macraei Hook. \& Arn. & Asteraceae & $\operatorname{Ar}$ & $E$ & $381 \mathrm{~b}$ & $95 \mathrm{c}$ & & $5(1 ; 30) c$ \\
\hline Cryptocarya alba (Molina) Looser & Lauraceae & A & $E$ & & & $10(38 ; 207) \mathrm{b}$ & \\
\hline Escallonia leucantha J. Remy & Escalloniaceae & $\mathrm{Ar}$ & $\mathrm{N}$ & & & $10(4 ; 50) \mathrm{b}$ & $5(7 ; 120) c$ \\
\hline $\begin{array}{l}\text { Escallonia pulverulenta (Ruiz \& Pav.) } \\
\text { Pers. }\end{array}$ & Escalloniaceae & $\mathrm{Ar}$ & $E$ & & $190 \mathrm{bc}$ & $238(9 ; 82) b$ & $214(9 ; 87) \mathrm{c}$ \\
\hline Eucalyptus globulus Labill. & Myrtaceae & A & 1 & $*$ & & & $5(11 ; 365) c$ \\
\hline $\begin{array}{l}\text { Gaultheria insana (Molina) D.J. } \\
\text { Middleton }\end{array}$ & Ericaceae & $\mathrm{Ar}$ & $\mathrm{N}$ & & $190 \mathrm{bc}$ & $1.286(3 ; 23) b$ & $95(5 ; 25) c$ \\
\hline Gevuina avellana Molina & Proteaceae & A & $\mathrm{N}$ & & & & $10(7 ; 112) \mathrm{c}$ \\
\hline $\begin{array}{l}\text { Gochnatia foliosa (D. Don) D. Don ex } \\
\text { Hook. \& Arn. }\end{array}$ & Asteraceae & $\mathrm{Ar}$ & $E$ & & & & $29(12 ; 80) c$ \\
\hline Lithrea caustica (Molina) Hook. \& Arn. & Anacardiaceae & A & $E$ & & & & $14(5 ; 69) c$ \\
\hline Lomatia hirsuta (Lam.) Diels & Proteaceae & A & $E$ & & & $114(6 ; 69) b$ & $19(5 ; 55) c$ \\
\hline Luma apiculata (DC.) Burret & Myrtaceae & A & $\mathrm{N}$ & & & $48(21 ; 33) b$ & $48(16 ; 71) c$ \\
\hline Nothofagus glauca (Phil.) Krasser & Nothofagaceae & A & $E$ & $286 \mathrm{~b}$ & & $971(6 ; 159) b$ & $650(7 ; 157) \mathrm{bc}$ \\
\hline Persea lingue (Ruiz \& Pav.) Nees & Lauraceae & A & $\mathrm{N}$ & & & $19(16 ; 79) b$ & $38(9 ; 95) c$ \\
\hline Podanthus mitiqui Lindl. & Asteraceae & $\mathrm{Ar}$ & $E$ & $95 \mathrm{~b}$ & $143 c$ & $171(5 ; 56) b$ & \\
\hline Quillaja saponaria Molina & Quillajaceae & A & $\mathrm{N}$ & & & $29(22 ; 119) \mathrm{b}$ & \\
\hline Ribes trilobum Meyen & Grossulariaceae & $\mathrm{Ar}$ & $E$ & $381 \mathrm{~b}$ & $1.143 b$ & $57(2 ; 27) b$ & $19(5 ; 68) c$ \\
\hline Schinus polygamus (Cav.) Cabrera & Anacardiaceae & $\mathrm{Ar}$ & $\mathrm{N}$ & & & $19(7 ; 150) \mathrm{b}$ & \\
\hline Teucrium bicolor Sm. & Lamiaceae & $\mathrm{Ar}$ & $E$ & $95 \mathrm{~b}$ & $1.000 \mathrm{~b}$ & $124(6 ; 25) b$ & $48(15 ; 34) c$ \\
\hline Ugni molinae Turcz. & Myrtaceae & $\mathrm{Ar}$ & $\mathrm{N}$ & & $333 \mathrm{bc}$ & $6.752(6 ; 23) a$ & $1.376(6 ; 25) a$ \\
\hline Viola portalesia Gay & Violaceae & Sa & $E$ & & & $229(4 ; 14) b$ & \\
\hline
\end{tabular}

$\mathrm{Ha}=$ Hábito ( $\mathrm{A}=$ Árbol; $\mathrm{Ar}=$ Arbusto; $\mathrm{Sa}=$ Subarbusto); Of = Origen fitogeográfico ( $\mathrm{N}=\mathrm{Nativa}$; $\mathrm{E}=$ Endémica de Chile; $\mathrm{I}=\mathrm{Introducida)}$;

* Especie encontrada fuera de muestreo. 


\section{AGRADECIMIENTOS}

Se agradece al proyecto FPA 7-RE-004-2018 "Reforestación Nativay Desarrollo Forestal Sustentable" yal apoyo de Suzanne Wylie y Cristóbal Rebolledo de Fundación Reforestemos. A la ayuda de Nicolás García en la determinación de algunas especies y de Alejandro Carrasco y Álvaro González por la colaboración en terreno.

\section{REFERENCIAS}

AGRIMED. 2017. Atlas agroclimático de Chile. Estado actual y tendencias del clima. Tomo III: regiones de Valparaíso, Metropolitana, O'Higgins y del Maule. FIA y Universidad de Chile, Santiago. 208 pp.

Amigo, J., San Martín, J., García Quintanilla, L. 2000. Estudio fitosociológico de los bosques de Nothofagus glauca (Phil.) Krasser del Centro-Sur de Chile. Phytocoenologia 30(2): 193-221.

Bustamante, R.O., Serey, I., Pickett, S.T.A. 2003. Forest fragmentation, plant regeneration and invasion processes in Central Chile. In: Bradshaw, G., Marquet, P., Mooney, M. (eds.), How landscapes change: Human disturbance and ecosystem fragmentation in the Americas, pp. 145160, Springer-Verlag, New York, USA.

Castillo, M., Garfias, R., Julio, G., González, L. 2012. Análisis de grandes incendios forestales en la vegetación nativa de Chile. Interciencia 37(11): 796-804.

Castillo, M., Garfias, R., Bown, H., Ruiz, F., Mahias, E., Hernández, C. 2017. Informe técnico. Análisis de las afectaciones postfuego en el Predio Pantanillos de la Universidad de Chile y recomendaciones de restauración. Informe Técnico. Facultad de Ciencias Forestales y de la Conservación de la Naturaleza, Universidad de Chile, Santiago. 4 pp.

CONAF. 2017. Análisis del impacto de los incendios forestales ocurridos en enero y febrero de 2017 sobre los ecosistemas naturales presentes entre las regiones de Coquimbo y Los Ríos de Chile. Informe Técnico. Ed. Santiago. $51 \mathrm{pp}$.

Echeverría, C., Coomes, D., Salas, J., Reyes-Benayas, J.M., Lara, A., Newton, A. 2006. Rapid deforestation and fragmentation of Chilean Temperate forests. Biological Conservation 130: 481-494.

Gómez, P., Hahn, S. 2017. Regeneración posincendio de plantas leñosas en plantaciones de Pinus radiata D. Don, zona costera, Región del Maule, Chile central. Gayana Botánica 74(2): 302-306.

Gómez, P., Hahn, S., San Martín, J. 2009. Estructura y composición florística de un matorral bajo plantaciones de Pinus radiata

D. Don en Chile central. Gayana Botánica 66(2): 256-268.

Gómez, P., Bustamante, R., San Martín, J., Hahn, S. 2011. Estructura poblacional de Pinus radiata D. Don en fragmentos de Bosque Maulino en Chile central. Gayana Botánica 68(1): 97-101.

Gómez-González, S., Paula, S., Cavieres, L.A., Pausas, J.G. 2017. Postfire responses of the woody flora of Central Chile: Insights from a germination experiment. PLoS ONE 12(7): e0180661.

González, M.E., Lara, A., Urrutia, R., Bosnich, J. 2011. Cambio climático y su impacto potencial en la ocurrencia de incendios forestales en la zona centro-sur de Chile $\left(33^{\circ}\right.$ $\left.42^{\circ} \mathrm{S}\right)$. Bosque 32(3): 215-219.

Keeley, J.E. 2012. Fire in mediterranean climate ecosystems A comparative overview. Israel Journal of Ecology \& Evolution 58: 123-135.

Litton, C.M., Santelices, R. 2002. Early post-fire succession in a Nothofagus glauca forest in the Coastal Cordillera of south-central Chile. International Journal of Wildland Fire 11: 115-125.

McDonald, P.M., Laacke, R.J. 1990. Pinus radiata D. Don. In: Burns, R.M., Honkala, B.H. (eds.), Silvics of North America: 1. Conifers, pp. 433-441, Forest Service, United States Department of Agriculture, Washington DC, USA.

Montenegro, G., Ginocchio, R., Segura, A., Keely, J.E., Gómez, M. 2004. Fire regimes and vegetation responses in two Mediterranean-climate regions. Revista Chilena de Historia Natural 77: 455-464.

Richardson, D.M., Brown, P.J. 1986. Invasion of mesic mountain fynbos by Pinus radiata. South African Journal of Botany 52(6): 529-536.

Rodríguez, R., Marticorena, C., Alarcón, D., Baeza, C., Cavieres, L., Finot, V.L., Fuentes, N., Kiessling, A., Mihoc, M., Pauchard, A., Ruiz, E., Sánchez, P., Marticorena, A. 2018. Catálogo de las plantas vasculares de Chile. Gayana Botánica 75(1): 1-430.

Rubilar, R., Blevins, L., Toro, J., Vita, A., Muñoz, F. 2008. Early performance of Pinus radiata plantations to weed control and fertilization on metamorphic soils of the Coastal Range, Maule Region, Chile. Bosque 29(1): 74-84.

San Martín, J., Donoso, C. 1995. Estructura florística e impacto antrópico en el bosque maulino de Chile. En: Armesto, J.J., Villagrán, C., Arroyo, M.K. (eds.), Ecología de los bosques nativos de Chile, pp. 153-168, Editorial Universitaria, Santiago, Chile.

Seidl, R., Thom, D., Kautz, M., Martin-Benito, D., Peltoniemi, M., Vacchiano, G., Wild, J., Ascoli, D., Petr, M., Honkaniemi, J., Lexer, M.J., Trotsiuk, V., Mairota, P., Svoboda, M., Fabrika, 
M., Nagel, T.A., Reyer, C.P.O. 2017. Forest disturbances under climate change. Nature Climate Change 7: 395402.

Thom, D., Seidl, R. 2016. Natural disturbance impacts on ecosystem services and biodiversity in temperate and boreal forests. Biological Reviews 91: 760-781.

Úbeda, X., Sarricolea, P. 2016. Wildfires in Chile: A review. Global and Planetary Change 146: 152-161.
Valencia, D., Saavedra, J., Brull, J., Santelices, R. 2018. Severidad del daño causado por los incendios forestales en los bosques remanentes de Nothofagus alessandrii Espinosa en la Región del Maule de Chile. Gayana Botánica 75(1): 531-534.

Williams, M.C. 2007. The ecological impacts of invasive Pinus radiata in eucalypt vegetation: pattern and process. Thesis Doctor of Philosophy. School of Biological Sciences, University of Sidney. Sydney, Australia. 226 pp.

Received: 13.08.2018

Accepted: 09.05.2019 